\title{
Regional variation of left ventricular transit time in normal ventricles and those with abnormalities of contraction
}

\author{
S WALTON, N J G BROWN, P H JARRITT, P J ELL, R W EMANUEL \\ From the Department of Cardiology and Institute of Nuclear Medicine, Middlesex Hospital and Medical School, \\ London
}

SUMMARY Thirty-three subjects were studied by first pass radionuclide angiography. The extrapolated monoexponential tracer washout slopes from septal, lateral, and apical segments of the left ventricle were measured.

In 13 subjects with normal left ventricles, as assessed by ejection fraction estimation and phase analysis, there was no difference between septal and lateral segments but, in each case, apical washout was significantly prolonged.

In 20 subjects with abnormal ventricles washouts were, in general, slower than those of the normal ventricles. Apical washout was still the slowest but the difference between base and apex was less pronounced. In contrast to the normal ventricles, differences appeared between septal and lateral segments.

The left ventricle is a poor mixing chamber. Pools of relative stasis exist at the apex of both normal and abnormal ventricles and within the body of the chamber in the latter.

That systolic emptying of the left ventricle is incomplete is widely appreciated, but the factors that determine which portion of the end-diastolic volume remains behind and the fate of this residual blood over the next and subsequent cardiac cycles are poorly understood.

This study uses regional differences in the time taken for a radionuclide bolus to pass through the left ventricle to detect areas of relative stasis within the chamber and compares normal ventricles with those with abnormalities of contraction in this respect.

\section{Subjects and method}

Thirty-three subjects referred for investigation of chest pain were studied by first pass radionuclide angiocardiography. $750 \mathrm{MBq}$ of ${ }^{99} \mathrm{~m}$ technetium as pertechnetate were injected as a bolus into a medial antecubital fossa vein and flushed with $20 \mathrm{ml}$ saline. The first pass of the tracer through the central circulation was detected by a General Electric IGE 400T

This work was supported by a grant from the Sir Jules Thorne Charitable Trust. Accepted for publication 9 September 1982 wide field of view gamma camera, fitted with a high sensitivity, parallel hole collimator and positioned over the precordium in the $45^{\circ}$ left anterior oblique projection with 15 to $20^{\circ}$ caudal tilt. Data were recorded for $\mathbf{4 0}$ seconds after injection in list mode by an on line Informatek Simis 3 computer along with electrocardiographic $R$ wave markers and $10 \mathrm{~ms}$ time markers.

By summing data from six to 12 cardiac cycles during the left heart phase of bolus transit a 16 frame representative cine cycle, that is a series of images representing a single average cardiac cycle, was produced. These images were used to determine ejection fraction from background corrected count rate changes within a manually assigned left ventricular region of interest and also for phase analysis. ${ }^{12}$

Phase analysis is a technique in which regions of the radioactively labelled blood pool are colour coded according to the timing, relative to the $R$ wave of the electrocardiogram, of the cyclic rise and fall of activity which occurs with each heart beat and which reflects filling and emptying. Normally contracting segments can be differentiated by their "phase shift" from regions with delayed or paradoxical emptying. Region of interest assignment is more accurate and regional 


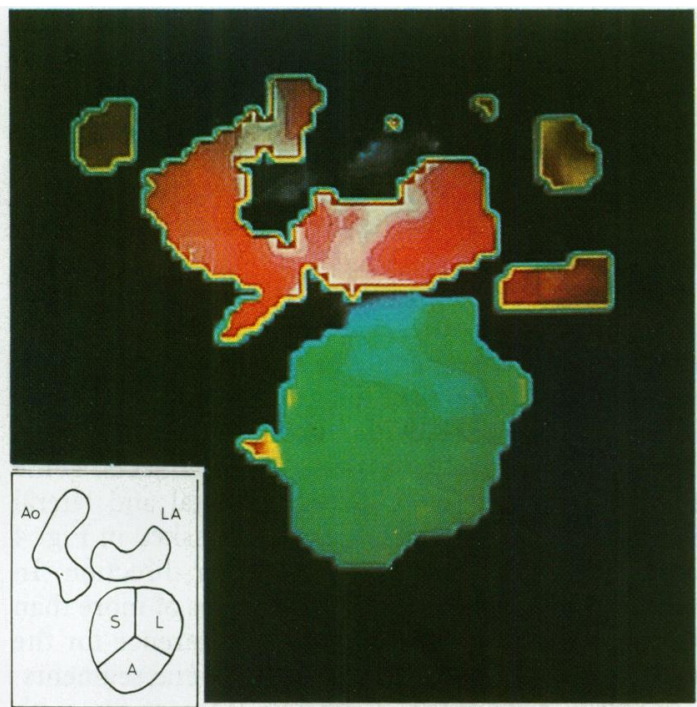

Fig. 1

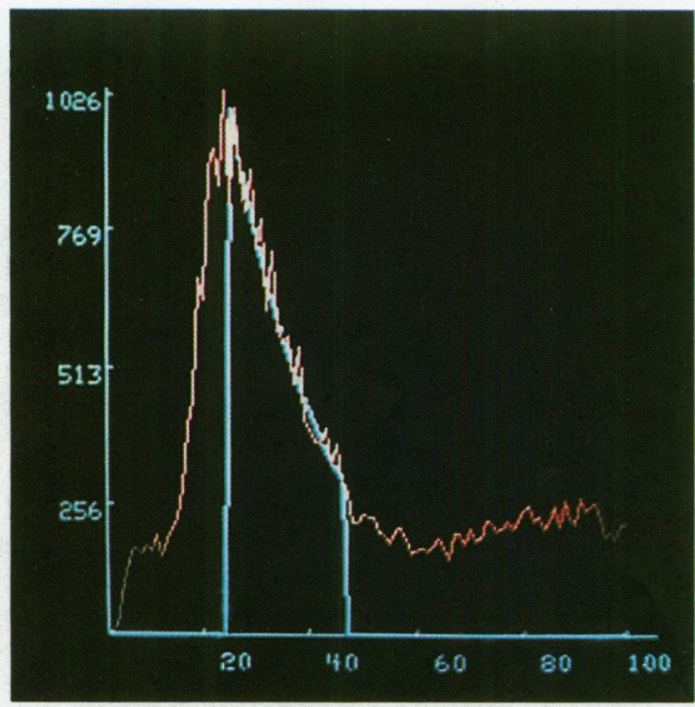

Fig. 3

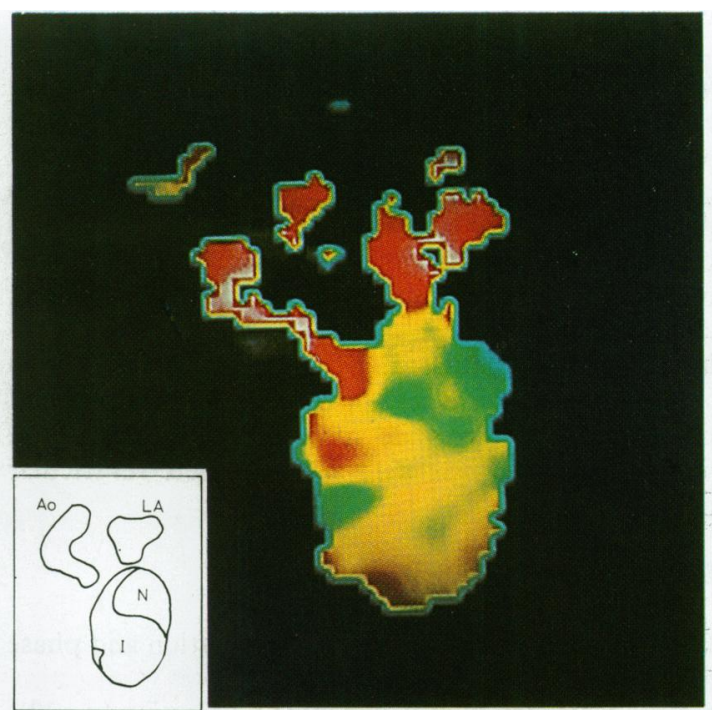

Fig. 2

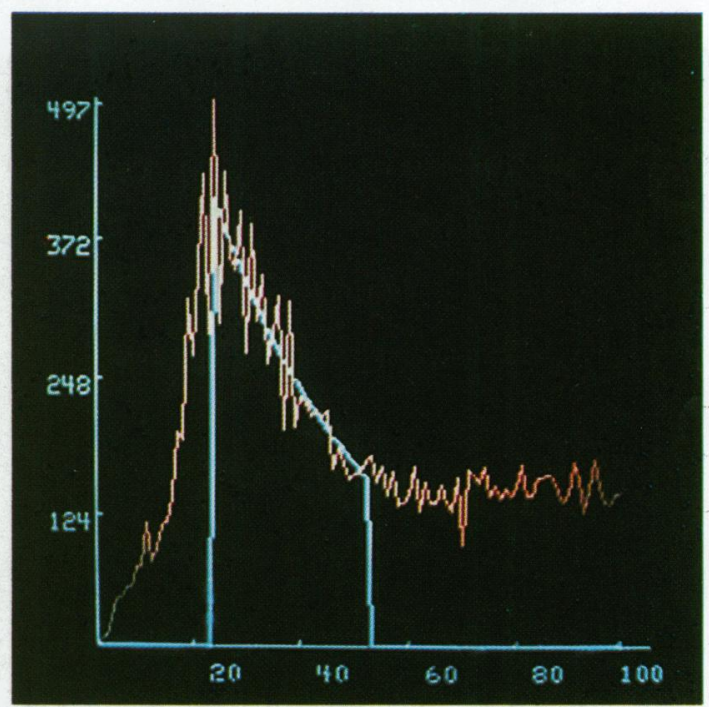

Fig. 4

Fig. 1 Normal phase image. L-lateral, $S$-septal, A-apical segments of the left ventricle. Ao, aorta; LA, left atrium. The ventricle is a region of low phase and coloured blue or green while the left atrium and aorta are regions of high phase and are coloured red and purple.

Fig. 2 Abnormal phase image. $N$, normally emptying region of the left ventricle. $I$, region of late emptying corresponding to infarction consequent upon occlusion of right and left anterior descending coronary arteries. Note the intermediate and high phase values (yellow and red, respectively) within the infarcted region. Ao, aorta; LA, left atrium.

Fig. 3 Septal activity time curve, normal subject. Raw data (red) and superimposed single exponential washout extrapolation. Washout slope 0.56.

Fig. 4 Apical activity time curve, normal subject. Raw data (red) and superimposed single exponential washout extrapolation (blue). Washout slope 0.36. 
ventricular disease can be detected and quantified.

Three $120^{\circ}$ segments were outlined on the phase image: septal, lateral, and apical. Mean phase was estimated for each region and compared to a previously established ${ }^{3}$ normal range.

An activity time curve of bolus transit, with 100 points and a temporal resolution of $400 \mathrm{~ms}$ was also produced for each segment. The curves were corrected for recirculation by extrapolation, assuming single exponential decay, and the slope of the extrapolated washout calculated.

The results of ejection fraction estimation and phase analysis were used to define two groups, one with normal and one with abnormal left ventricular contraction.

\section{Results}

On the basis of ejection fraction estimation and phase analysis two groups were defined.

Group 1: Normal left ventricular contraction $(n=13)$. Ejection fractions above $50 \%$ and normal phase images.

Group 2: Abnormal left ventricular contraction $(\mathrm{n}=20)$. Either ejection fractions below $50 \%$ or abnormal phase images or both.

Fig. 1 and 2 show phase images from a normal and an abnormal subject, respectively. Fig. 3 and 4 show

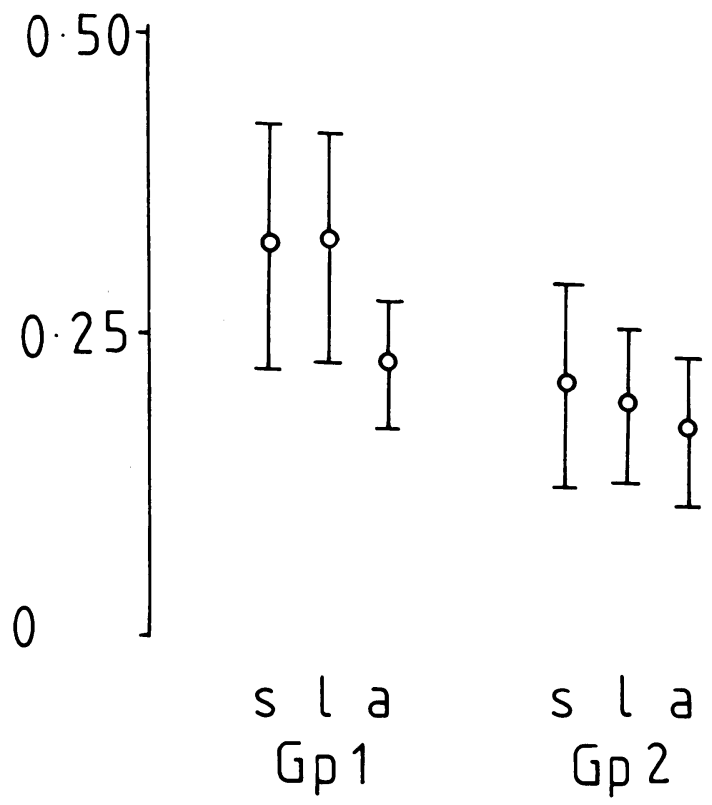

Fig. 5 Washout slopes. s-septal, l-lateral, a-apical segments. Gp 1, group 1; Gp 2, group 2. Group means and standard deviations. representative segmental activity time curves from a normal subject.

Washout slopes, expressed as group means and standard deviations, are shown in Fig. 5. Significant differences were between septal and apical $(p<0.005)$ and lateral and apical $(\mathrm{p}<0.005)$ segments in group 1 and between septal and apical segments in group 2 $(0.025>p<0.01)$.

Comparison of individual septal, lateral, and apical washouts showed differences of $1 \pm 2 \%$ (mean \pm SD) between septal and lateral and $30 \pm 13 \%$ between septal and apical segments of the group 1 subjects. These differences compare with those of $13 \pm 11 \%$ and $23 \pm 20 \%$, respectively, for the group 2 subjects. Some of the differences found between septal and lateral segments in the group 2 subjects are masked in Fig. 4 because the difference can be in either direction. In fact, 13 group 2 subjects had differences of more than $5 \%$, a value 2 SD above the mean difference for the group 1 subjects, between septal and lateral segments. Conversely, four group 2 subjects (four of five with ejection fractions below 20\%) had apical-septal differences $2 \mathrm{SD}$ below the mean value for the group 1 subjects.

\section{Discussion}

Despite the importance of such knowledge to our understanding of cardiac mechanics and to the $\stackrel{\square}{\square}$ interpretation of the results of a number of clinical $\overrightarrow{\overrightarrow{0}}$ and research procedures, little is known about mixing 3 in the human left ventricle. The only evidence that mixing is incomplete comes from the discrepancy between dye dilution and angiographic estimates of left ventricular volume. 45

The canine left ventricle has been more extensively investigated and several studies ${ }^{6-12}$ have produced evidence of poor mixing. They show that the passage of various tracers through the left ventricle is not uniform, apical transit being prolonged relative to that of the base.

After bolus intravenous injection of a radioactive $\frac{D}{2}$ tracer it is thoroughly mixed with blood by the time that it reaches the left heart. If mixing is complete in $\stackrel{N}{\circ}$ the left ventricle the tracer should pass uniformly through all regions of the chamber.

The left ventricular activity time curve comprises a single low frequency component, reflecting bolus transit through the chamber, and superimposed high $\stackrel{0}{=}$ frequency fluctuations, corresponding to filling and $\stackrel{\infty}{\Phi}$ emptying of the ventricle with each cardiac cycle. At a temporal resolution of $400 \mathrm{~ms}$ the curves predominantly reflect the low frequency component (Fig. 3). Furthermore, flow variations at heart rate frequencies $\frac{\rho}{\mathbb{Q}}$ do not significantly affect the overall shape, and there- $\varrho$ fore measurements of bolus transit, of indicator dilu- 
tion curves recorded in the central circulation. ${ }^{13}$ This is not to say that tracer redistribution within the left ventricle does not occur with each cardiac cycle, merely that it is not reflected in the overall shape of the curve. There are a number of ways to characterise the shape of indicator dilution curves, and that of the washout slope has been widely used.

In the normal ventricles there was no difference between septal and lateral washout, but apical washout was significantly prolonged relative to that of the two basal segments. In the abnormal ventricles apical washout was again the slowest though basal-apical differences were somewhat smaller and that between apical and lateral segments did not reach statistical significance.

Slow apical washout can be explained by the arrangement of both mitral and aortic valves at the base of the ventricle. Early in bolus transit increasing concentrations of tracer are being delivered to the base through the mitral valve. Thus, though cardiac contraction moves non-labelled blood from the apex towards the base, it is labelled blood that is preferentially ejected and the non-labelled blood is preferentially retained. As the tracer concentration in the blood passing through the mitral valve rises to a peak, mixing will occur and labelled blood will reach the apex. The situation is then reversed. The concentration of tracer at the base will fall and the labelled blood at the apex will be preferentially retained while non-labelled blood is expelled. This effect is often apparent on the computer display of a first pass study. Tracer enters the base of both ventricles and is expelled. Only after several subsequent cardiac cycles does tracer percolate down to the apex of the chamber.

Changes in chamber size and shape in the abnormal ventricles mean that septal, lateral, and apical segments do not necessarily correspond to the same regions of normal ventricles.

Differences appear in the abnormal ventricles between septal and lateral segments which were not present in the normal ventricles. These differences presumably reflect relative stasis in the proximity of abnormally contracting segments. Paradoxically, in ventricles with low values of ejection fraction differences between base and apex become minimal. These results probably reflect slow, uniform diffusion of tracer through a dilated, almost akinetic portion of the chamber.

The results of this study are totally in accord with the previous studies on the canine ventricle mentioned above. Nevertheless, the possibility of the differences being artefactual must be considered. One possible source of such error would be contamination of the apical curves by activity from a chamber with late transit. Early recirculation of tracer from the coronary bed occurs in the right ventricle, but in the left anterior oblique projection this is unlikely to represent a significant problem. The activity time curves must contain a contribution from myocardial blood flow ${ }^{14}$ but this must be small and there is no reason to expect such great apical-basal differences in myocardial flow in normal subjects. Tracer passing down the descending aorta remains a possible source of contamination, but it would be unlikely to produce such a pronounced and constant effect.

Though the derivation of indicator dilution curves from individual chambers is standard practice during first pass radionuclide angiocardiography, compartmental analysis within chambers is not. Each segmental activity time curve is the sum of the activity time curves from all display elements within that segment. As we envisage a continuous distribution of transit times between base and apex, deriving from the relatively slow percolation first of labelled and then unlabelled blood from base to apex, there will be a significant degree of inhomogeneity within each segment. Furthermore, there is, over a number of cardiac cycles, considerable interaction between the segments. These factors are theoretical objections to the use of a single exponential washout extrapolation, but in practice there is a very close approximation to such a model, as shown in Fig. 3 and 4. Certainly, the results cannot be explained in terms of curve fitting artefact.

\section{Conclusion}

Even in health, the left ventricle is a poor mixing chamber. There is an apical pool of relative stasis compared to the basal regions. The results of this study are consistent with the hypothesis that the left ventricle operates on a "last in-first out" basis.

In ventricles damaged by coronary artery disease the same phenomenon is found but with additional regions of slow transit within the body of the chamber which are presumably related to abnormal contraction patterns.

Techniques requiring the assumption that mixing within the ventricle is complete, such as first pass radionuclide angiocardiography and much dye dilution work, may well be invalid.

\section{References}

1 Bossuyt A, Deconink F, Lepoidre R, Joncker M. The temporal Fourier transform applied to the functional image. In: Di Paola R, Kahn E, eds. Information processing in medical imaging. Proceedings of 6th International Conference. Paris: INSERM, 1980: 88: 397-408.

2 Links JM, Douglass KH, Wagner HN Jr. Patterns of ventricular emptying by Fourier analysis of gated blood-pool studies. F Nucl Med 1980; 21: 978-82. 
3 Walton S, Yiannikas J, Jarritt PH, Brown NJG, Swanton RH, Ell PJ. Phasic abnormalities of left ventricular emptying in coronary artery disease. $\mathrm{Br}$ Heart $\mathcal{F} 1981$; 46: 245-53.

4 Rolett EL, Sherman H, Gorlin R. Measurement of left ventricular volume by thermodilution: an appraisal of technical errors. I Appl Physiol 1964; 19: 1164-74.

5 Carleton RA, Bowyer AF, Graettinger JS. Overestimation of left ventricular volume by the indicator dilution technique. Circ Res 1966; 18: 248-56.

6 Irisawa H, Wilson MF, Rushmer RF. Left ventricle as a mixing chamber. Circ Res 1960; 8: 183-7.

7 Swan HJC, Beck W. Ventricular nonmixing as a source of error in the estimation of ventricular volume by the indicator-dilution technique. Circ Res 1960; 8: 989-98.

8 Petrle M, Avasthey P. Some problems in the estimation of the end-diastolic volume of the ventricle by dye dilution techniques. Cardiovasc Res 1968; 2: 193-8.

9 Bartle SH, Sanmarco ME. Measurement of left ventricular volume by biplane angiocardiography and indicator washout techniques: a comparison in the canine heart. Circ Res 1966; 19: 295-306.

10 Pavek E, Pavek K, Boska D. Mixing and observation errors in indicator dilution studies. $\mathcal{f}$ Appl Physiol 1970; 28: $733-40$.

11 Boyle J III. Indicator mixing in the left heart and reexamination of mean circulation time. $\mathcal{F}$ Appl Physiol 1974; 37: $541-6$.

12 Castellana FS, Snapinn SM, Tam SY, Case RB. Inlet and intrachamber concentration distributions in tracer studies of the canine central circulation and their relation to the isotope dilution residue function. Circ Res 1980; 47: 10-20.

13 Bassingthwaighte JB, Knopp TJ, Anderson DU. Flow estimation by indicator dilution (bolus injection). Reduction of errors due to time averaged sampling during unsteady flow. Circ Res 1970; 27: 277-91.

14 Mena I, Thomsen P, Bennett LR. Determination of coronary blood flow index by external scintillation detection. F Nucl Med 1963; 4: 259-76.

Requests for reprints to Dr S Walton, Department of Cardiology, Middlesex Hospital, Mortimer Street, London WIN 8AA. 